Engineering Optimization

Vol. 00, No. 00, Month 20XX, 1-17

\title{
Experiments with hybrid Bernstein global optimization algorithm for the OPF problem in power systems
}

\author{
Bhagyesh V. Patil ${ }^{a}{ }^{*}$, L.P.M.I. Sampath ${ }^{b}$, Ashok Krishan ${ }^{c}$, \\ J. M. Maciejowski ${ }^{d}$, K. V. Ling ${ }^{c}$, H. B. Gooi ${ }^{c}$ \\ ${ }^{a}$ Cambridge Centre for Advanced Research and Education in Singapore (CARES) \\ ${ }^{b}$ Interdisciplinary Graduate School, Nanyang Technological University, Singapore \\ ${ }^{c}$ School of Electrical 83 Electronic Engineering, Nanyang Technological University, Singapore \\ ${ }^{d}$ Department of Engineering, University of Cambridge, United Kingdom
}

(Received 00 Month 20XX; final version received 00 Month 20XX)

\begin{abstract}
This paper presents an algorithm based on the Bernstein form of polynomials for solving the optimal power flow (OPF) problem in electrical power networks. The proposed algorithm combines local and global optimization methods and is therefore referred to as a 'hybrid' Bernstein algorithm in the context of this work. The proposed algorithm is a branch-andbound $(\mathrm{B} E \mathrm{~B} B)$ procedure wherein a local search method is used to obtain a good upper bound on the global minimum at each branching node. Subsequently, the Bernstein form of polynomials is used to obtain a lower bound on the global minimum. The performance of the proposed algorithm is compared with the previously reported Bernstein algorithm to demonstrate its efficacy in terms of the chosen performance metrics. Furthermore, the proposed algorithm is tested by solving the OPF problem for several benchmark IEEE power system examples and its performance is compared with generic global optimization solvers such as BARON and COUENNE. The test results demonstrate that the algorithm HBBB delivers satisfactory performance in terms of solution optimality.
\end{abstract}

Keywords: Bernstein polynomials; Global optimization; Power systems; Optimal power flow; Network optimization; Nonconvex problems.

\section{Nomenclature}

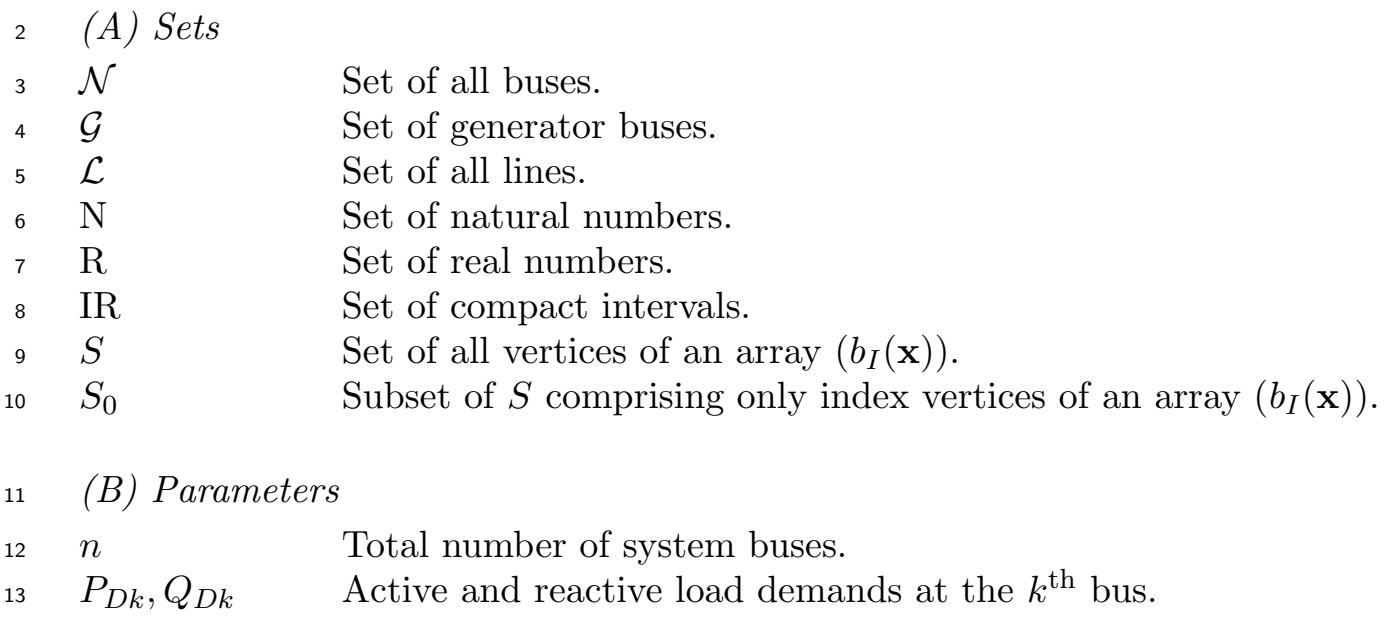

*Corresponding author. Email: bvpatil@ntu.edu.sg 


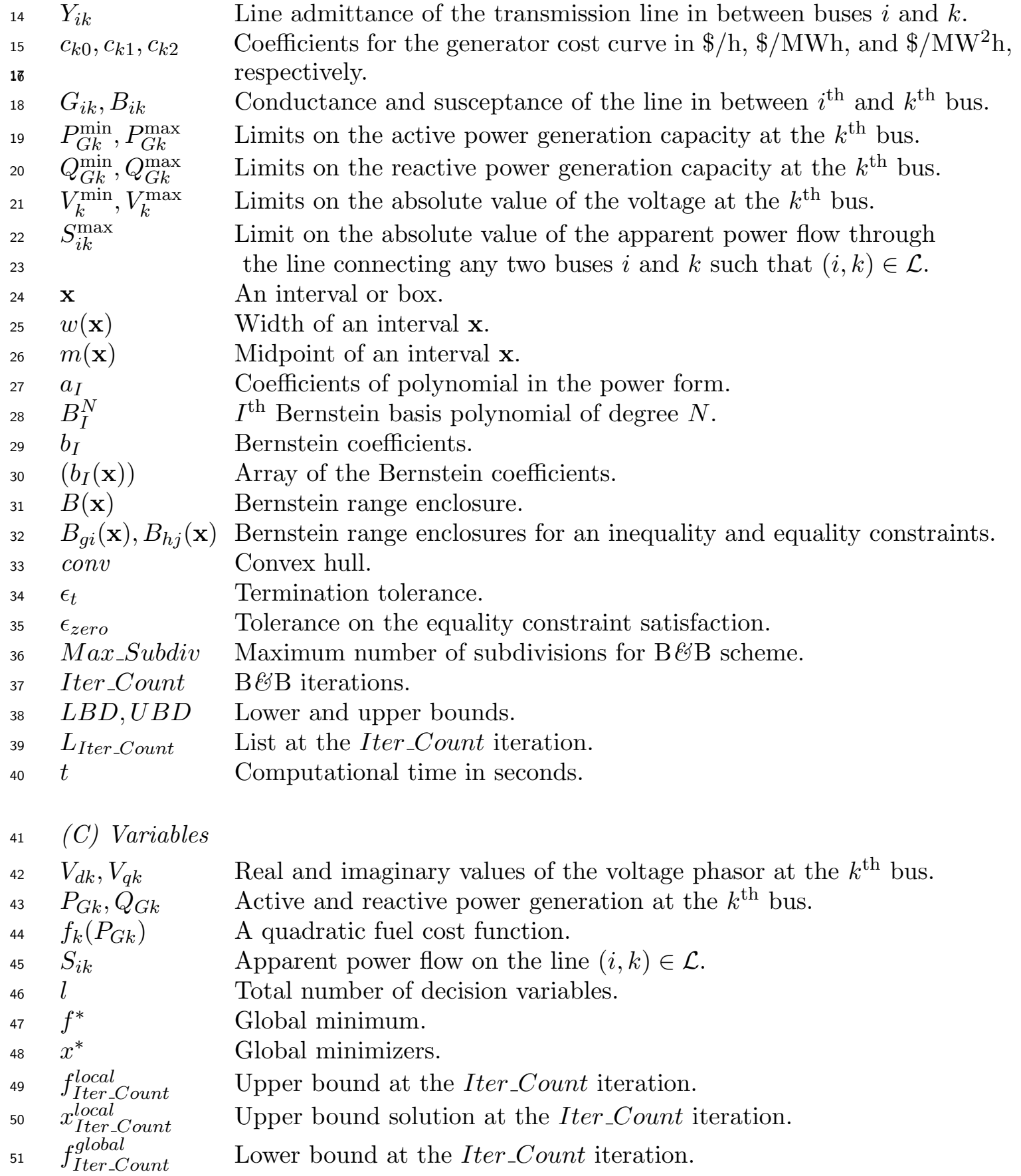

Line admittance of the transmission line in between buses $i$ and $k$. respectively.

Limits on the active power generation capacity at the $k^{\text {th }}$ bus.

Limits on the reactive power generation capacity at the $k^{\text {th }}$ bus.

Limit on the absolute value of the apparent power flow through

the line connecting any two buses $i$ and $k$ such that $(i, k) \in \mathcal{L}$.

Midpoint of an interval $\mathbf{x}$.

Coefficients of polynomial in the power form

$I^{\text {th }}$ Bernstein basis polynomial of degree $N$.

ients.

intein coefficients.

Convex hull.

Tolerance on the equality constraint satisfaction.

Maximum number of subdivisions for B $\mathscr{E} B$ scheme.

$\mathrm{B} \mathscr{E} \mathrm{B}$ iterations.

List at the Iter_Count iteration.

Computational time in seconds.

Lower bound at the Iter_Count iteration.

\section{Introduction}

Numerical optimization algorithms play a vital role in ensuring the stable and reliable operation of modern electric power systems (Kundur (1994); Capitanescu (2016)). Among other applications, optimization algorithms are used in network expansion planning problems and generator scheduling problems. The OPF problem is one such well studied problem in the power systems community. The OPF problem aims at optimizing network operations by finding optimal operating points for the electric generators in the system. It achieves this by minimizing the total power generation cost subject to certain network constraints. Some of these constraints include generator active and reactive 
power generation limits, bus voltage magnitudes, and network constraints. An excellent recent survey about the OPF problem can be found in Capitanescu (2016).

The complexity involved in the OPF problem is mainly two-fold: (i) the size of realworld OPF problems for which a direct solution approach is prohibitive due to memory and computational time limitations and (ii) nonconvex problem structure resulting from highly nonlinear power balance equations, which demand good global optimization procedures to determine the optimal operating points for the generators. In this work, we primarily focus on addressing (ii) with specific application to benchmark IEEE power system examples.

Several deterministic solution approaches have been proposed for solving the OPF problem. Prominent among these are sequential linear and quadratic programming, Lagrangian relaxation, and interior-point methods (see, for instance Phan and Kalagnanam (2014); Momoh, El-Hawary, and Adapa (1999a); Momoh, El-Hawary, and Adapa (1999b); Gopalakrishnan et al. (2012)). However, as noted above, the OPF problem is nonconvex in nature with multiple equilibrium points (cf. Bukhsh et al. (2013)). Consequently, the aforementioned solution approaches, which typically rely on a 'convexity' assumption of the optimization problem, may fail to find the good optimal solution in practice. In addition to the aforementioned solution approaches, semidefinite programming (SDP) relaxation is another popular method which is widely used for solving the OPF problem (Bai et al. (2008)). However, the exactness of the SDP relaxation can only be guaranteed for radial networks (see, for instance, Kocuk, Dey, and Xu. A. Sun (2016)). Other research directions in the context of the OPF problem are based on the development of convex envelopes (Zhijun, Hou, and Chen (2015)) and decomposition based global optimization methods ( $\mathrm{Li}$ and $\mathrm{Li}(2016)$ ).

Similarly, in the past decade, a number of non-deterministic solution approaches have also been investigated for solving OPF problems. A few examples of such approaches are ant colony optimization (Soares et al. (2011)), genetic algorithm (Todorovski and Rajicic (2006)), differential evolution (A. A. Abou El Ela, Abido, and Spea (2010); Shaheen, El-Sehiemy, and Farrag (2016)), particle swarm optimization (Abido (2002); Vaisakh and Srinivas (2011); Mohamed et al. (2017)), simulated annealing (Roa-Sepulveda and Pavez-Lazo (2003)), bacterial foraging algorithm (Edward et al. (2013)), and imperialist competitive algorithm (Ghasemi et al. (2014a); Ghasemi et al. (2014b); Ghasemi et al. (2015)). A detailed survey of deterministic and non-deterministic solution approaches for solving the OPF problem can be found in Frank, Steponavice, and Rebennack (2012a) and Frank, Steponavice, and Rebennack (2012b).

We note that the last two decades have witnessed the emergence of interval form based B EB B has emerged as a promising framework to solve nonconvex optimization problems (Vaidyanathan and M. El-Halwagi (1996); Hansen and Walster (2005)). This is evident from the seminal work on $\alpha \mathrm{BB}$ relaxation by Adjiman, Androulakis, and Floudas (1998) which had yielded BEB implementations, such as BARON (Tawarmalani and Sahinidis 2005) and COUENNE (Belotti et al. 2009). The impressive performances of BARON and COUENNE on a wide variety of optimization problems has been well documented. In recent times, various modifications of the aforementioned $\mathrm{B} \mathscr{E} \mathrm{B}$ implementations have also been reported in the literature (see, work reported by Grimstad and Sandnes (2016), Gerard, Kppe, and Louveaux (2017), Castro (2017), and references therein). This has motivated us to investigate an alternative interval form based Bernstein global optimization algorithm to solve the polynomial OPF problem.

This work explores the well-known Bernstein form of polynomials (Ratschek and Rokne (1988)), and uses several attractive 'geometrical' properties associated with the Bernstein form (refer to Section 3.1). Optimization procedures based on the Bernstein form, also called Bernstein global optimization algorithms, have shown good promise in solving hard (nonconvex) nonlinear programming (NLP) and mixed-integer nonlinear programming 
(MINLP) problems (see, for instance, Nataraj and Arounassalame (2011); Patil, Nataraj, and Bhartiya (2012)). Recently, a Bernstein global optimization algorithm was also proposed to solve the OPF problem for small power networks (see Patil et al. (2016)). As such, we believe that further investigations in the context of the OPF problem using the Bernstein global optimization approach seems to be a promising research direction.

In this work, we propose a hybrid ${ }^{1}$ branch-and-bound $(\mathrm{B} \mathscr{E} \mathrm{B})$ algorithmic scheme. Specifically, we use the Bernstein polynomial form in conjunction with a local NLP solving technique to form a new hybrid Bernstein global optimization algorithm(hereinafter referred to as algorithm HBBB). The algorithm HBBB uses an iterative subdivision procedure in a $\mathrm{B} \mathscr{E} \mathrm{B}$ scheme, wherein a series of upper and lower bounding subproblems are solved at each node of the BEBB tree. We obtain the upper bound using MATLAB's 'fmincon' as a local NLP solver and the lower bound using the minimum Bernstein coefficient value (see Theorem 3.1). Furthermore, we follow the principle of interval analysis, wherein iterative subdivisions are performed at each step of a BEB scheme. This enables the $\mathrm{B} \mathscr{E} \mathrm{B}$ scheme to converge the upper and lower bounds within a user-specified accuracy. The overall schematic of the proposed approach is depicted in Figure 1.

We first show with a simple nonlinear optimization problem the effectiveness of the algorithm HBBB over the previously reported Bernstein algorithm in (Nataraj and Arounassalame (2011)), and the state-of-the-art BARON solver. The performance comparison is made on the basis of the number of boxes processed, and the computational time required to locate the correct global solution. Subsequently, we assess the scalability and performance of the algorithm HBBB over the OPF problem for the several benchmark IEEE power system network examples. The performance of the proposed algorithm HBBB is compared with the generic global optimization solvers BARON (Tawarmalani and Sahinidis (2005)) and COUENNE (Belotti et al. (2009)).

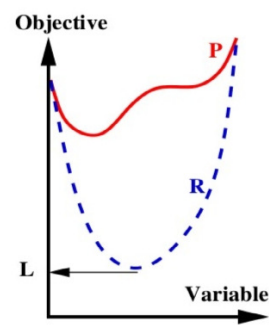

(a) Lower bounding process

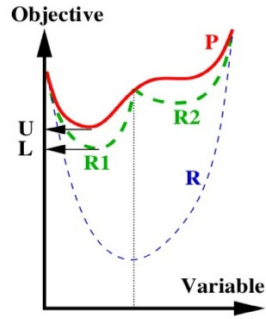

(c) Convergence using subdivision

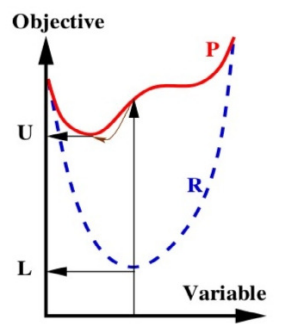

(b) Upper bounding process

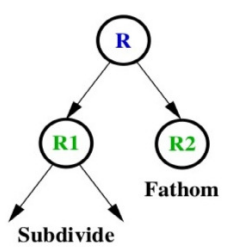

(d) B\&B scheme

Figure 1. The hybrid Bernstein BEB scheme illustrating the lower (L) and upper (U) bounding processes followed by subdivision. $\mathrm{P}$ represents the original (nonconvex) problem, whose global minimum is to be sought and $\mathrm{R}$ is the convex relaxation obtained (in our case using the Bernstein polynomial form).

\footnotetext{
${ }^{1}$ The word hybrid in this context means that our algorithm is a combination of local and global optimization methods. To the best of the authors' knowledge, this is the first work which explores the use of local solving techniques for the early pruning of nodes in a BEB B tree in the context of Bernstein global optimization algorithms.
} 
The remainder of this paper is organized as follow. The classical OPF formulation for the power network first is first introduced in Section 2. Next, the Bernstein polynomial form is briefly introduced in Section 3. This is followed by a description of our proposed algorithm HBBB in Section 4. The results from numerical studies performed with our algorithm HBBB on some benchmark IEEE power system network examples are reported in Section 5. The results of the numerical studies are also compared with those obtained using well established global optimization solvers in Section 5. Finally, some concluding remarks and directions for future research are given in Section 6.

\section{Optimal power flow problem}

In this section, we briefly present the classical OPF formulation along the lines of Molzahn et al. (2013) which is in terms of the rectangular power and voltage co-ordinates. The objective of the OPF problem is to minimize the cost of real power generation. The problem is subject to constraints such as the power balance, satisfaction of bus voltage limits, active and reactive power generation limits, and line-flow limits.

Consider an $n$-bus power system, where $\mathcal{N}=\{1,2, \ldots, n\}$ represents the set of all buses; $\mathcal{G}$ represents the set of generator buses and $\mathcal{L}$ represents the set of all lines. Let $P_{D k}$ and $Q_{D k}$ represent the active and reactive power demands respectively at each bus $k \in \mathcal{N}$. Let $V_{k}=V_{d k}+\mathrm{j} V_{q k}$ represent the voltage phasor in rectangular coordinates at each bus $k \in \mathcal{N}$. Let $P_{G k}$ and $Q_{G k}$ represent the active and reactive power generations respectively at each generator bus $k \in \mathcal{G}$. Let $S_{i k}$ represent the apparent power flow and $Y_{i k}=G_{i k}+\mathrm{j} B_{i k}$ denote the line admittance of the line $(i, k) \in \mathcal{L}$ respectively.

The quadratic fuel cost function associated with each generator $k \in \mathcal{G}$ representing a $\$ / \mathrm{h}$ operating cost is given below.

$$
f_{k}\left(P_{G k}\right)=c_{k 2} P_{G k}^{2}+c_{k 1} P_{G k}+c_{k 0} \quad \forall k \in \mathcal{G}
$$

Then, the classical OPF optimization problem can be stated as follows:

$$
\min _{P_{G k}, Q_{G k}, V_{d k}, V_{q k}} f=\sum_{k \in \mathcal{G}} f_{k}\left(P_{G k}\right)
$$

subject to

$$
\begin{aligned}
& P_{G k}-P_{D k}=V_{d k} \sum_{i=1}^{n}\left(G_{i k} V_{d i}-B_{i k} V_{q i}\right)+V_{q k} \sum_{i=1}^{n}\left(B_{i k} V_{d i}+G_{i k} V_{q i}\right) \quad \forall k \in \mathcal{N} \\
& Q_{G k}-Q_{D k}=V_{d k} \sum_{i=1}^{n}\left(-B_{i k} V_{d i}-G_{i k} V_{q i}\right)+V_{q k} \sum_{i=1}^{n}\left(G_{i k} V_{d i}-B_{i k} V_{q i}\right) \quad \forall k \in \mathcal{N} \\
& P_{G k}^{\min } \leqslant P_{G k} \leqslant P_{G k}^{\max } \quad \forall k \in \mathcal{G} \\
& Q_{G k}^{\min } \leqslant Q_{G k} \leqslant Q_{G k}^{\max } \quad \forall k \in \mathcal{G} \\
& \left(V_{k}^{\min }\right)^{2} \leqslant V_{d k}^{2}+V_{q k}^{2} \leqslant\left(V_{k}^{\max }\right)^{2} \quad \forall k \in \mathcal{N} \\
& P_{k i}=G_{i k}\left(V_{d k}^{2}+V_{q k}^{2}\right)-G_{i k}\left(V_{d k} V_{d i}+V_{q k} V_{q i}\right)+B_{i k}\left(V_{d i} V_{q k}-V_{d k} V_{q i}\right) \quad \forall k \in \mathcal{N} \\
& Q_{k i}=B_{i k}\left(V_{d k}^{2}+V_{q k}^{2}\right)-G_{i k}\left(V_{d i} V_{q k}-V_{d k} V_{q i}\right)-B_{i k}\left(V_{d k} V_{d i}+V_{q k} V_{q i}\right) \quad \forall k \in \mathcal{N} \\
& \sqrt{P_{k i}^{2}+Q_{k i}^{2}} \leqslant S_{k i}^{\max } \quad \forall(i, k) \in \mathcal{L}
\end{aligned}
$$


The objective function (2) is the minimization of the total operating cost of the system. Equations (3) and (4) are the real and reactive power balance constraints at each bus $k$. Equations (3) and (4) are formulated considering the Kirchoff's laws of power flow through branches attached to buses. Active and reactive power generation capability margins are considered in (5) and (6) respectively. Equations (7) and (10) represent the voltage security margins and the line apparent power flow capacities respectively.

Remark 1 We note that the constraints (3)-(4) possess multilinear terms in the real and imaginary voltage components. Hence, the OPF problem turns out to be a nonconvex nonlinear programming (NLP) problem, albeit polynomial in nature (i.e., (2)-(4) are always polynomials in the power form shown in (11)).

\section{The Bernstein polynomial approach}

In this section, we introduce some notions related to interval analysis and the theory pertaining to the Bernstein form of polynomials presented in Patil, Nataraj, and Bhartiya (2012). Interested readers may also refer to Ratschek and Rokne (1988) and Moore, Kearfott, and Cloud (2009) for more details about this topic.

\subsection{Bernstein form}

Let $l \in \mathrm{N}$ be the number of variables and $x=\left(x_{1}, x_{2}, \ldots, x_{l}\right) \in \mathrm{R}^{l}$. A multi-index $I$ is defined as $I=\left(i_{1}, i_{2}, \ldots, i_{l}\right) \in \mathrm{N}^{l}$ and the multi-power $x^{I}$ is defined as $x^{I}=\left(x_{1}^{i_{1}}, x_{2}^{i_{2}}, \ldots, x_{l}^{i_{l}}\right)$. Another multi-index $N$ is defined as $N=\left(n_{1}, n_{2}, \ldots, n_{l}\right)$. Inequalities $I \leq N$ for multiindices are meant component-wise. With $I=\left(i_{1}, \ldots, i_{r-1}, i_{r}, i_{r+1}, \ldots, i_{l}\right)$, we associate the index $I_{r, k}$ given by $I_{r, k}=\left(i_{1}, \ldots, i_{r-1}, i_{r+k}, i_{r+1}, \ldots, i_{l}\right)$, where $0 \leq i_{r+k} \leq n_{r}$. Also we write $\left(\begin{array}{c}N \\ I\end{array}\right)$ for $\left(\begin{array}{c}n_{1} \\ i_{1}\end{array}\right) \cdots\left(\begin{array}{c}n_{l} \\ i_{l}\end{array}\right)$ and $(N / I)$ for $\left(n_{1} / i_{1}, n_{2} / i_{2}, \ldots, n_{l} / i_{l}\right)$ provided that $0<i_{k}$, $k=1,2, \ldots, l$.

A real, bounded and closed interval $\mathbf{x}$ is defined as follows:

$$
\mathbf{x}=[\underline{x}, \bar{x}]:=[\inf \mathbf{x}, \sup \mathbf{x}] \in \mathrm{IR},
$$

where IR denotes the set of compact intervals. Let $w(\mathbf{x})$ denote the width of $\mathbf{x}$, that is $w(\mathbf{x}):=\bar{x}-\underline{x}$, and $m(\mathbf{x})$ denote the midpoint of $\mathbf{x}$, that is $m(\mathbf{x}):=(\bar{x}+\underline{x}) / 2$. For an $l$-dimensional interval vector or box $\mathbf{x}=\left(\mathbf{x}_{1}, \mathbf{x}_{2}, \ldots, \mathbf{x}_{l}\right) \in \mathrm{IR}^{l}$, the width of $\mathbf{x}$ is $w(\mathbf{x}):=\max \left(w\left(\mathbf{x}_{1}\right), w\left(\mathbf{x}_{2}\right), \ldots, w\left(\mathbf{x}_{l}\right)\right)$.

We can write an $l$-variate polynomial $p$ in the power form as shown below.

$$
p(x)=\sum_{I \leq N} a_{I} x^{I}, \quad x \in \mathrm{R}^{l},
$$

with $N$ being the degree of $p$. We expand a given multivariate polynomial $p$ into Bernstein polynomials to obtain the bounds for its range over an $l$-dimensional box $\mathbf{x}$. The $I^{\text {th }}$ Bernstein basis polynomial of degree $N$ is defined as follows:

$$
B_{I}^{N}(x)=B_{i_{1}}^{n_{1}}\left(x_{1}\right) \cdots B_{i_{l}}^{n_{l}}\left(x_{l}\right), \quad x \in \mathrm{R}^{l},
$$


where for $i_{j}=0,1, \ldots, n_{j}, j=1,2, \ldots, l$

$$
B_{i_{j}}^{n_{j}}\left(x_{j}\right)=\left(\begin{array}{c}
n_{j} \\
i_{j}
\end{array}\right) \frac{\left(x_{j}-\underline{x}_{j}\right)^{i_{j}}\left(\bar{x}_{j}-x_{j}\right)^{n_{j}-i_{j}}}{\left(\bar{x}_{j}-\underline{x}_{j}\right)^{n_{j}}} .
$$

The Bernstein coefficients $b_{I}(\mathbf{x})$ of $p$ over the box $\mathbf{x}$ are given by the following equation:

$$
b_{I}(\mathbf{x})=\sum_{J \leq I} \frac{\left(\begin{array}{c}
I \\
J
\end{array}\right)}{\left(\begin{array}{c}
N \\
J
\end{array}\right)} w(\mathbf{x})^{J} \sum_{K \leq J}\left(\begin{array}{c}
K \\
J
\end{array}\right)(\inf \mathbf{x})^{K-J} a_{K}, \quad I \leq N
$$

The Bernstein form of a multivariate polynomial $p$ is defined by

$$
p(x)=\sum_{I \leq N} b_{I}(\mathbf{x}) B_{I}^{N}(x)
$$
THEOREM 3.1 (Range enclosure property) Let $p$ be a poly
$\bar{p}(\mathbf{x})$ denote the range of $p$ on a given box $\mathbf{x} \in \mathrm{IR}^{l}$. Then,

$$
\bar{p}(\mathbf{x}) \subseteq B(\mathbf{x}):=\left[\min \left(b_{I}(\mathbf{x})\right)_{I \in S}, \max \left(b_{I}(\mathbf{x})\right)_{I \in S}\right] .
$$

Proof: See Garloff (1993).

Remark 2 The above theorem states that the minimum and maximum coefficients of the array $\left(b_{I}(\mathbf{x})\right)_{I \in S}$ provide lower and upper bounds for the range. This forms the Bernstein range enclosure defined by $B(\mathbf{x})$ in (16).

Lemma 3.2 (Convex hull property) Let $\left(b_{I}(\mathbf{x})\right)$ be an array of Bernstein coefficients for a polynomial $p(x)$ on a given box $\mathbf{x} \in \mathrm{IR}^{l}$. Then, the following property holds:

$$
\operatorname{conv}(x, p(x)) \subseteq\left(I / N, b_{I}(\mathbf{x}): I \in S\right),
$$

where conv $(x, p(x))$ denotes the convex hull of $p$.

Remark 3 The above lemma states that the range of $p(x)$ is contained in the convex hull of the control points $\left(I / N,\left(b_{I}\right)\right)$. Figure 2 illustrates this fact, wherein the polynomial function is represented as $p(x)$ and Bernstein coefficients are denoted as $b_{0}, b_{1}, b_{2}, b_{3}, b_{4}$, and $b_{5}$. The dotted lines in Figure 2 define the convex hull. Furthermore, this Bernstein range enclosure can be successively sharpened by the continuous domain subdivision procedure. This is illustrated in Figure 3. 


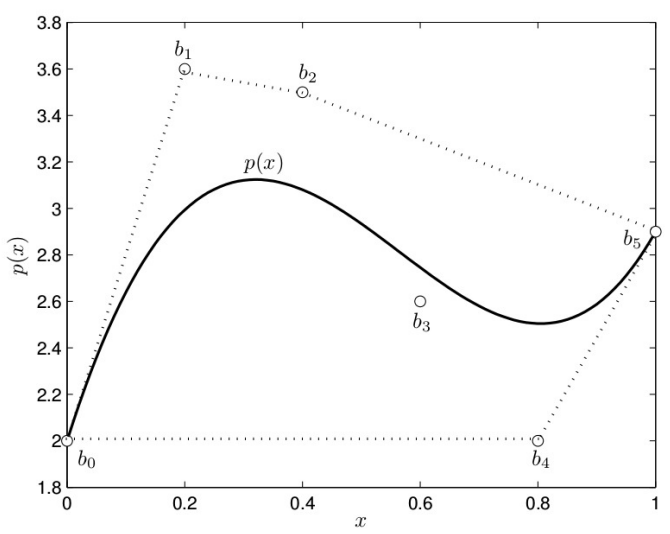

Figure 2. A polynomial function $p$, its Bernstein coefficients and the convex hull over a box $\mathbf{x}=[0,1]$.

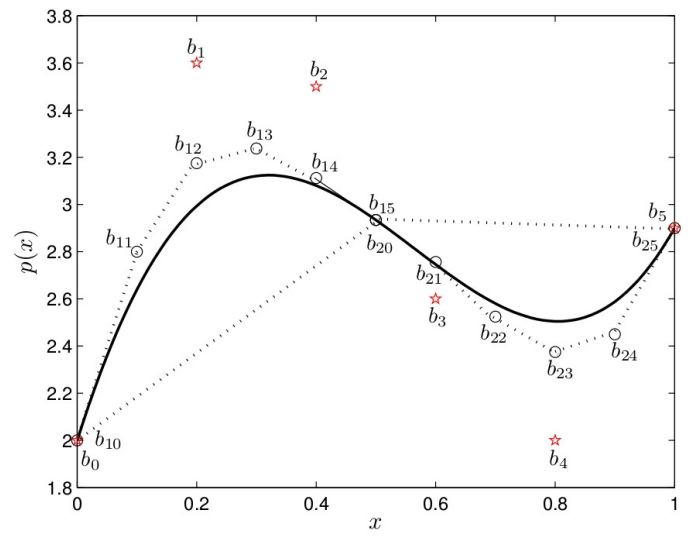

Figure 3. Improvement in the range enclosure of $p$ with a subdivision of the original box $\mathbf{x}=[0,1]$. $\left(b_{10}, b_{11}, b_{12}, b_{13}, b_{14}, b_{15}\right)$, and $\left(b_{20}, b_{21}, b_{22}, b_{23}, b_{24}, b_{25}\right)$ are the Bernstein coefficients over $\mathbf{x}_{1}=[0,0.5]$ and $\mathbf{x}_{2}=[0.5,1]$, respectively.

The following properties follow immediately from Theorem 3.1.

Lemma 3.3 Let $B(\mathbf{x})$ be the Bernstein range enclosure for a polynomial $p(x)$ on a given box $\mathrm{x} \in \mathrm{IR}^{l}$. Then, the following properties hold

(1) $B(\mathbf{x}) \leq 0 \Rightarrow p(x) \leq 0$ for all $x \in \mathbf{x}$.

(2) $B(\mathbf{x})>0 \Rightarrow p(x)>0$ for all $x \in \mathbf{x}$.

(3) $0 \notin B(\mathbf{x}) \Rightarrow p(x) \neq 0$ for all $x \in \mathbf{x}$.

(4) $B(\mathbf{x}) \subseteq[-\epsilon, \epsilon] \Rightarrow p(x) \in[-\epsilon, \epsilon]$ for all $x \in \mathbf{x}$, where $\epsilon>0$.

\section{Hybrid Bernstein global optimization algorithm}

In this section, we outline the proposed algorithm HBBB to solve polynomial NLP problems. We first briefly describe the algorithm. Subsequently, we demonstrate the strength of the algorithm HBBB over the previously reported Bernstein algorithm (Nataraj and Arounassalame (2011)) on a nonlinear optimization problem. Furthermore, with the optimization problem, we also demonstrate the merits of the algorithm HBBB over the BARON solver. Finally, in Section 5, the algorithm HBBB is used to determine the optimal solution (global minimum and minimizers) of the OPF problem (2)-(4) described in Section 2

Briefly, the algorithm works as follows. At the outset, for the original problem, a 
feasible solution (from the search box $\mathbf{x}_{\text {Iter_Count }}$ ) is computed using a local search method. The obtained minimum is called a feasible upper bound $(U B D)$. Next, a valid lower bound $(L B D)$ on the optimal objective function value is obtained using the minimum Bernstein coefficient value. After establishing the upper and lower bounds on the global minimum, we refine them. This is accomplished by successively subdividing the initial box $\mathbf{x}_{\text {Iter_Count }}$ at the midpoint along the longest side, resulting in two smaller boxes $\left(\mathbf{x}_{\text {Iter_Count }, 1}, \mathbf{x}_{\text {Iter_Count }, 2}\right)$. This procedure generates a nonincreasing sequence for the upper bound and a nondecreasing sequence for the lower bound. Within a finite number of subdivisions, the gap between $U B D$ and $L B D$ shrinks to the termination accuracy $\epsilon_{t}$. Finally, the algorithm terminates with the current upper bounding solution as the global solution.

Algorithm hybrid Bernstein: $\left[f^{*}, x^{*}\right]=\operatorname{HBBB}\left(f, g, h, \mathbf{x}, \epsilon_{t}, \epsilon_{\text {zero }}\right.$, Max_Subdiv $)$

Inputs: The objective function $(f)$ and constraints $(g, h)$ in the power form, the initial search box $\mathbf{x}$, parameter $\epsilon_{t}$ as the termination accuracy, tolerance parameter $\epsilon_{z e r o}$ to which the equality constraints are to be satisfied, and Max_Subdiv as the maximum number of subdivisions to be performed to locate the global solution.

Outputs: A global minimum $f^{*}$ and global minimizers $x^{*}$ over a box $\mathbf{x}$.

\section{BEGIN Algorithm}

(1) $\{$ Initialization $\}$

Set Iter_Count $\longleftarrow 0$, Subdiv_No $\longleftarrow 0, L B D \leftarrow-\infty, U B D \leftarrow \infty, \mathbf{x}_{\text {Iter_Count }} \leftarrow \mathbf{x}$, $\mathbf{x}_{\text {Iter_Count }}^{c} \leftarrow m\left(\mathbf{x}_{\text {Iter_Count }}\right), L_{\text {Iter_Count }} \leftarrow\{\}$.

(2) $\{$ Upper bound computation $\}$

Solve OPF (2)-(4) over $\mathbf{x}_{\text {Iter_Count }}$ using a local search method. We use $\mathbf{x}_{\text {Iter_Count }}^{c}$ as an initial point to start the optimization for a local NLP solver. Denote the obtained

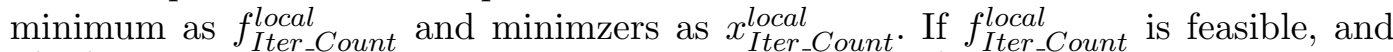
$f_{\text {Iter_Count }}^{\text {local }}<U B D$, then update $U B D$ as $U B D \leftarrow f_{\text {Iter_Count }}^{\text {local }}$.

(3) $\{$ Subdivision $\}$

Subdivide the current box $\mathbf{x}_{\text {Iter_Count }}$ into two smaller subboxes

(a) Subdiv_No $\leftarrow$ Subdiv_No +1 .

(b) Choose a coordinate direction $\lambda$ parallel to which $\mathbf{x}_{\text {Iter_Count }, 1} \times \cdots \times \mathbf{x}_{\text {Iter_Count }, l}$ has an edge of maximum length, that is $\lambda \in\left\{i: w(\mathbf{x}):=w\left(\mathbf{x}_{i}\right), i=1, \ldots, l\right\}$.

(c) Bisect $\mathbf{x}_{\text {Iter_Count }}$ normal to direction $\lambda$, getting boxes $\mathbf{x}_{\text {Iter_Count, } 1}$ and $\mathbf{x}_{\text {Iter_Count }, 2}$, such that $\mathbf{x}_{\text {Iter_Count }}=\mathbf{x}_{\text {Iter_Count }, 1} \cup \mathbf{x}_{\text {Iter_Count }, 2}$.

(4) $\{$ Lower bound computation $\}$

for $k=1,2$

(a) Find the Bernstein coefficients and the corresponding Bernstein range enclosure of the objective function $(f)$ over $\mathbf{x}_{\text {Iter_Count, } k}$ as $b_{0}\left(\mathbf{x}_{\text {Iter_Count }, k}\right)$ and $B_{0}\left(\mathbf{x}_{\text {Iter_Count, } k}\right)$, respectively.

(b) Set $f_{\text {Iteral }}^{\text {glount }}:=\min B_{o}\left(\mathbf{x}_{\text {Iter_Count }, k}\right)$.

(c) If $f_{\text {Iter_Count }}^{\text {global }}>U B D$, then go to substep (g).

(d) for $i=1,2, \ldots, m$

(i) Find the Bernstein coefficients and the corresponding Bernstein range enclosure of the inequality constraint polynomial $\left(g_{i}\right)$ over $\mathbf{x}_{\text {Iter_Count }, k}$ as $b_{g i}\left(\mathbf{x}_{\text {Iter_Count }, k}\right)$ and $B_{g i}\left(\mathbf{x}_{\text {Iter_Count }, k}\right)$, respectively.

(ii) If $B_{g i}\left(\mathbf{x}_{\text {Iter_Count }, k}\right)>0$, then go to substep (g).

(iii) If $B_{\text {gi }}\left(\mathbf{x}_{\text {Iter_Count }, k}\right) \leq 0$, then go to substep (e)

(e) for $j=1,2, \ldots, n$ 
(i) Find the Bernstein coefficients and the corresponding Bernstein range enclosure of the equality constraint polynomial $\left(h_{j}\right)$ over $\mathbf{x}_{\text {Iter_Count }, k}$ as $b_{h j}\left(\mathbf{x}_{\text {Iter_Count }, k}\right)$ and $B_{h j}\left(\mathbf{x}_{\text {Iter_Count }, k}\right)$, respectively.

(ii) If $0 \notin B_{h j}\left(\mathbf{x}_{\text {Iter_Count }, k}\right)$ then go to substep (g).

(iii) If $B_{h j}\left(\mathbf{x}_{\text {Iter_Count }, k}\right) \subseteq\left[-\epsilon_{\text {zero }}, \epsilon_{\text {zero }}\right]$ then go to substep (f).

(f) Enter $\left(\mathbf{x}_{\text {Iter_Count }, k}, f_{\text {Iter_Count }}^{\text {global }}\right)$ into the list $\mathcal{L}_{\text {Iter_Count }}$ such that the second members of all the items of the list do not decrease.

(g) end (of $k$-loop).

(5) \{Update iteration counter and lower bound

(a) Set Iter_Count $\leftarrow$ Iter_Count +1 .

(b) Update LBD to the minimum of the second entries over all the items in $\mathcal{L}_{\text {Iter_Count }}$. Similarly, fetch the first entry corresponding to this minimum and denote it as $\mathbf{x}_{\text {Iter_Count }}$. Also compute $\mathbf{x}_{\text {Iter_Count }}^{c}$ as $\mathbf{x}_{\text {Iter_Count }}^{c} \leftarrow m\left(\mathbf{x}_{\text {Iter_Count }}\right)$.

(6) $\{$ Termination condition $\}$ If $S u b d i v \_N o<M a x_{-} S u b d i v$ or $U B D-L B D>\epsilon_{t}$, then go to step 2. Else go to step 7.

(7) $\{$ Compute global solution\}

Return the global minimum and global minimizers as $f^{*} \leftarrow U B D$, and $x^{*} \leftarrow$ $x_{\text {Iter_Count }}^{\text {local }}$, respectively.

\section{END Algorithm}

Remark 4 The algorithm HBBB follows a classical subdivision procedure for the original box $\mathbf{x}$. As such, the feasible region for $\mathbf{x}$ shrinks with each iteration. Furthermore, the objective function value is a function of $\mathbf{x}$. Hence, the sequence of upper and lower bounds converge in the limit within a finite number of iterations (cf. Ratschek and Rokne (1988)).

Remark 5 The subdivision of $\mathbf{x}$ aids in raising the lower bound (computed using the Bernstein form) of the objective function value (cf. Patil, Nataraj, and Bhartiya (2012)), thereby speeding up the convergence of the algorithm HBBB.

THEOREM 4.1 The algorithm HBBB based on the upper and lower bounding schemes converges to the global optimal solution.

Proof: The algorithm HBBB is both bound consistent (see Remark 4), and bound improving (see Remark 5). Hence, it is also convergent (Tawarmalani and Sahinidis (2002), Li and Sun (2010)).

Demonstrative Example: The strength of the algorithm HBBB is now demonstrated with a nonlinear optimization problem adapted from (Lebbah, Michel, and Rueher (2007)). We first demonstrate the strength of the algorithm HBBB with the previously reported Bernstein algorithm from Nataraj and Arounassalame (2011) in terms of the number of subdivisions and the computational time. Subsequently, we also demonstrate the merit of the algorithm HBBB in terms of the solution optimality when compared with the BARON solver.

Consider the following nonlinear optimization problem:

$$
\left.\begin{array}{cl}
\min _{x, y} & f=x \\
\text { subject to } & y-x^{2} \geq 0 \\
& y-x^{2}(x-2)+10^{-5} \leq 0 \\
& x \in \mathbf{x}=[-10,10], y \in \mathbf{y}=[-10,10]
\end{array}\right\}
$$




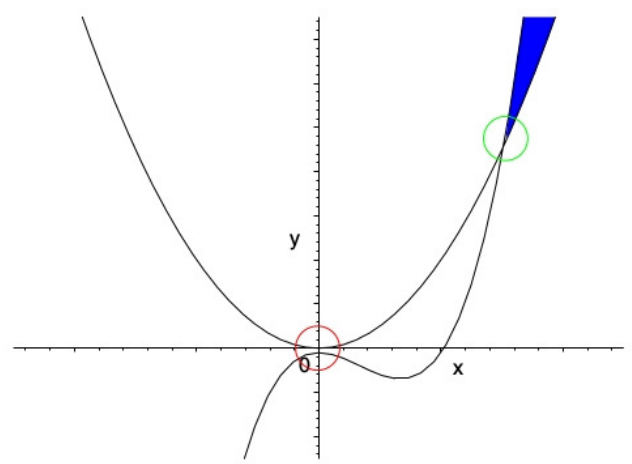

Figure 4. Geometrical representation of an optimization problem (P) (Lebbah, Michel, and Rueher (2007)).

Geometrically, this problem is shown in Figure 4. As pointed out by Lebbah, Michel, and Rueher (2007), the solution of the problem (P) lies in the neighborhood of the point $x \approx 3, y \approx 9$, with the global minimum as $f^{*} \approx 3$.

We observed that both the classical Bernstein algorithm and HBBB algorithm converged to the correct global solution reported by the Lebbah, Michel, and Rueher (2007) for the problem $(\mathrm{P})$. However, the algorithm HBBB was found to be superior in term of its performance during the global search process (cf. Table 1). The algorithm HBBB required approximately $66 \%$ fewer subdivisions, thereby reducing the computational time required by approximately $30 \%$.

Table 1. Performance comparison of the previously reported Bernstein algorithm in Nataraj and Arounassalame (2011) and the algorithm HBBB on the optimization problem (P).

\begin{tabular}{|c|c|c|}
\hline $\begin{array}{c}\text { Performance } \\
\text { metrics }\end{array}$ & $\begin{array}{c}\text { Bernstein algorithm } \\
\text { (Nataraj and Arounassalame (2011)) }\end{array}$ & HBBB \\
\hline \hline $\begin{array}{c}\text { Number of } \\
\text { subdivisions }\end{array}$ & 294 & 100 \\
\hline $\begin{array}{c}\text { Computational } \\
\text { time (seconds) }\end{array}$ & 2.53 & 1.78 \\
\hline
\end{tabular}

Furthermore, the problem (P) was solved using BARON with an optimality tolerance $10^{-12}$ (i.e. in GAMS, set optca $=10 \mathrm{E}-12$ ). BARON reported $f^{*}=0$ as the global minimum, and $x^{*}=0, y^{*}=0$ as the global minimizers. This successfully demonstrates the merit of the algorithm HBBB when compared with BARON for this particular optimization problem.

\section{Numerical results}

In this section, we report results from solving the OPF problem for several benchmark IEEE power system models with our hybrid Bernstein algorithm (HBBB). The benchmark IEEE power system models were adapted from Zimmerman, Murillo-Sanchez, and Thomas (2011). We analyze the results from two perspectives. First, the performance of algorithm HBBB for several test cases (3-, IEEE 9-, IEEE 14-, IEEE 30-, and IEEE 39-bus systems) is compared with the performance of general purpose global optimization solvers like BARON (Tawarmalani and Sahinidis (2005)) and COUENNE (Belotti et al. (2009)). Subsequently, we study the computational time growth of the algorithm 
Table 2. Comparison of the OPF cost $(2)\left(f^{*}\right.$, in $\left.\$ / \mathrm{h}\right)$ and computational time $(t$, in seconds) for benchmark IEEE test cases under different solution approaches.

\begin{tabular}{|c|c|c|c|c|c|}
\hline \multirow{2}{*}{$\begin{array}{c}\text { Test } \\
\text { case }\end{array}$} & Number of & Performance & \multicolumn{3}{|c|}{ Solver/Algorithm } \\
\cline { 4 - 6 } & decision variables & metric & BARON & COUENNE & HBBB $^{\dagger}$ \\
\hline \hline 3-bus & 12 & $f^{*}$ & 5703.52 & 5703.52 & 5703.52 \\
& & $t$ & 0.5 & 0.2 & 0.45 \\
\hline IEEE-9 & 24 & $f^{*}$ & 5296.68 & 5296.68 & 5296.68 \\
bus & & $t$ & 0.2 & 7.72 & 7.62 \\
\hline IEEE-14 & 38 & $f^{*}$ & 8081.53 & 8081.53 & 8081.53 \\
bus & & $t$ & 0.3 & 371.72 & 13.64 \\
\hline IEEE-30 & 72 & $f^{*}$ & 576.89 & 576.89 & 576.89 \\
bus & & $t$ & 396.93 & 1021.01 & 24.41 \\
\hline IEEE-39 & 98 & $f^{*}$ & 41864.18 & 41864.18 & 41864.18 \\
bus & & $t$ & 4.25 & 1026.83 & 48.75 \\
\hline
\end{tabular}




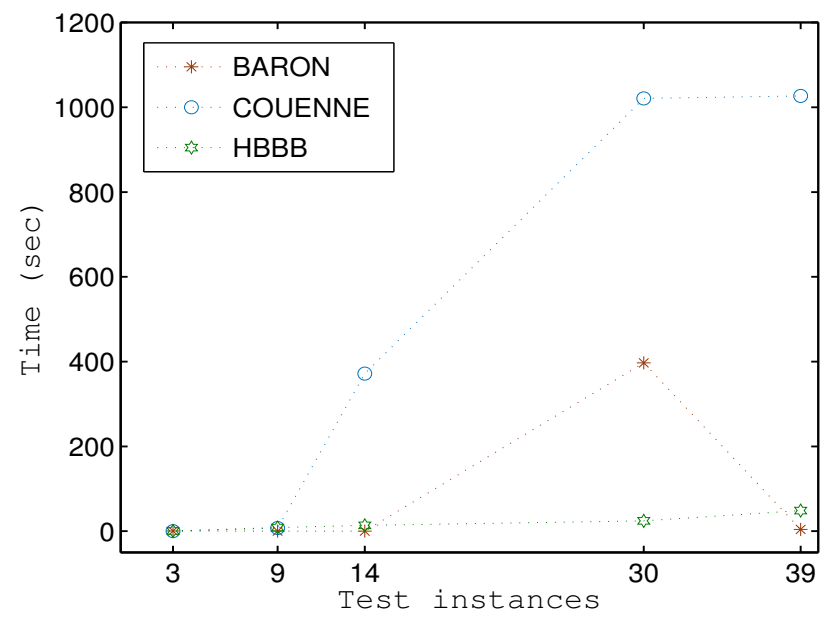

Figure 5. Computational time comparison for five test cases (3-, 9-, 14-, 30-, and 39-bus systems) solved using BARON, COUENNE and algorithm HBBB.

Table 3. Comparison of the cost function $(2)\left(f^{*}\right.$, in $\left.\$ / \mathrm{h}\right)$ and computational time ( $t$, in seconds) for benchmark IEEE test cases solved using the algorithm HBBB with increasing number of subdivisions and tightened termination accuracy $\epsilon_{t}$.

\begin{tabular}{|c|c|c|c|c|}
\hline \multirow{2}{*}{ Test } & Performance & \multicolumn{3}{|c|}{ Number of subdivisions $\left(\epsilon_{t}=10^{-8}\right)$} \\
\cline { 3 - 5 } instance & metric & 50 & 100 & 150 \\
\hline \hline 3 -bus & $f^{*}$ & 5703.52 & 5703.52 & 5703.52 \\
& $t$ & 3.44 & 7.95 & 17.90 \\
\hline IEEE-9 & $f^{*}$ & 5296.68 & 5296.68 & 5296.68 \\
bus & $t$ & 8.25 & 24.6 & 56.01 \\
\hline IEEE-14 & $f^{*}$ & 8081.53 & 8081.53 & 8081.53 \\
bus & $t$ & 32.43 & 52.47 & 79.15 \\
\hline IEEE-30 & $f^{*}$ & 576.89 & 576.89 & 576.89 \\
bus & $t$ & 52.42 & 125.27 & 322.82 \\
\hline IEEE-39 & $f^{*}$ & 41864.18 & 41864.18 & 41864.18 \\
bus & $t$ & 109.20 & 277.86 & 455.17 \\
\hline
\end{tabular}




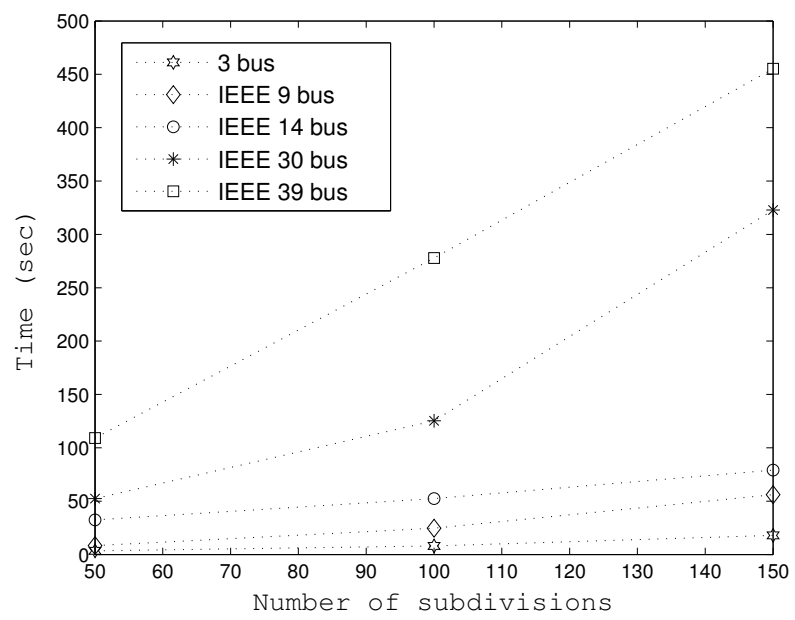

Figure 6. Computational time growth of the algorithm HBBB with increasing number of subdivisions (50, 100, 150) and tightened termination accuracy $\epsilon_{t}\left(10^{-8}\right)$.

Table 4. Equality constraint satisfaction at the optimal solution for five benchmark IEEE test cases solved using the algorithm HBBB under a tightened termination accuracy $\epsilon_{t}=10^{-8}$.

\begin{tabular}{|c|c|c|}
\hline \multirow{2}{*}{$\begin{array}{c}\text { Test } \\
\text { case }\end{array}$} & \multicolumn{2}{|c|}{ Equality constraint } \\
\cline { 2 - 3 } & Mean & Max \\
\hline \hline 3-bus & $7.04 \times 10^{-11}$ & $4.95 \times 10^{-10}$ \\
\hline IEEE-9 bus & $3.57 \times 10^{-12}$ & $1.74 \times 10^{-11}$ \\
\hline IEEE-14 bus & $8.97 \times 10^{-12}$ & $1.10 \times 10^{-10}$ \\
\hline IEEE-30 bus & $2.05 \times 10^{-14}$ & $1.39 \times 10^{-13}$ \\
\hline IEEE-39 bus & $1.19 \times 10^{-14}$ & $3.89 \times 10^{-13}$ \\
\hline
\end{tabular}

\section{Conclusions}

In this work, we presented a new BEB B scheme in the context of the OPF problem. Our scheme was based on the concept of sequential improvement in the upper and lower bounds of a BEB B tree. The interesting feature of our approach was the use of the Bernstein polynomial form in conjunction with a local search method (a 'hybrid' algorithm HBBB in our terminology). The efficacy of the algorithm HBBB was compared with the previously reported Bernstein algorithm using a nonlinear optimization instance. Furthermore, the same optimization instance was also used to demonstrate the merits of the algorithm HBBB over the BARON solver. Further, to ascertain the practicability of the algorithm HBBB, we tested it on several benchmark IEEE OPF instances and compared its performance with well established global optimization solvers such as BARON and COUENNE. In terms of computational time, the algorithm HBBB was slower than BARON except for one test instance (IEEE 30-bus system), where it performed exceptionally well. On the other hand, the algorithm HBBB was found to be faster than COUENNE for most test cases. We note that the algorithm HBBB was able to achieve the same optimality as BARON and COUENNE in terms of fuel cost for the OPF problem.

The work reported in this paper can be extended in the following directions: 
- The OPF problem in this work was restricted to small to medium-size power systems (to be precise, 3- to IEEE 39-bus). It is well-known that the size of OPF problem grows enormously with the size of the power system network. In such circumstances, distributed optimization algorithms hold a lot of promise. As such, we plan to extend the algorithm HBBB into a distributed framework.

- The problem formulation in this work considered a traditional fossil fuel based power generation network. The inclusion of intermittent renewable energy sources makes the OPF problem more challenging. In this scenario, solving the OPF problem requires the adoption of robust optimization procedures with chance constraints. In future, we plan to extend the algorithm HBBB to solve such problems.

\section{Acknowledgement}

This research is supported by the National Research Foundation, Prime Ministers Office, Singapore under its CREATE programme.

\section{References}

A. A. Abou El Ela, M. A. Abido, and S.R. Spea. 2010. "Optimal power flow using differential evolution algorithm." Electric Power Systems Research 80 (7): 878-885.

Abido, M. A. 2002. "Optimal power flow using particle swarm optimization." International Journal of Electrical Power Energy Systems 24 (7): 563-571.

Adjiman, C. S., I. P. Androulakis, and C. A. Floudas. 1998. "A global optimization method, $\alpha \mathrm{BB}$, for general twice-differentiable constrained NLPs-II. Implementation and computational results." Computers 83 Chemical Engineering 22 (9): 1159-1179.

Bai, X., H. Wei, K. Fujisawa, and Y. Wang. 2008. "Semidefinite programming for optimal power flow problems." International Journal of Electrical Power and Energy Systems 30 (6-7): 383392.

Belotti, P., J. Lee, L. Liberti, F. Margot, and A. Wächter. 2009. "Branching and bounds tightening techniques for non-convex MINLP." Optimization Methods and Software 24 (4-5): 597-634.

Bukhsh, W. A., A. Grothey, K. I. M. McKinnon, and P. A. Trodde. 2013. "Local solutions of the optimal power flow problem." IEEE Transactions on Power Systems 28 (4): 4780-4788.

Capitanescu, F. 2016. "Critical review of recent advances and future developments needed in AC optimal power flow." Electric Power Systems Research 136: 57-68.

Castro, P. M. 2017. "Spatial branch-and-bound algorithm for MIQCPs featuring multiparametric disaggregation." Optimization Methods and Software 32 (4): 719-737.

Edward, J. Belwin, N. Rajasekar, K. Sathiyasekar, N. Senthilnathan, and R. Sarjila. 2013. "An enhanced bacterial foraging algorithm approach for optimal power flow problem including FACTS devices considering system loadability." ISA Transactions 52 (5): 622-628.

Frank, S., I. Steponavice, and S. Rebennack. 2012a. "Optimal power flow: a bibliographic survey I." Energy Systems 3 (3): 221-258.

Frank, S., I. Steponavice, and S. Rebennack. 2012b. "Optimal power flow: a bibliographic survey II." Energy Systems 3 (3): 259-289.

Garloff, J. 1993. "The Bernstein algorithm." Interval Computations 2: 154-168.

Gerard, D., M. Kppe, and Q. Louveaux. 2017. "Guided dive for the spatial branch-and-bound." Journal of Global Optimization 68 (4): 685-711.

Ghasemi, Mojtaba, Sahand Ghavidel, Mohammad Mehdi Ghanbarian, Masihallah Gharibzadeh, and Ali Azizi Vahed. 2014a. "Multi-objective optimal power flow considering the cost, emission, voltage deviation and power losses using multi-objective modified imperialist competitive algorithm." Energy 78: 276-289.

Ghasemi, Mojtaba, Sahand Ghavidel, Mohammad Mehdi Ghanbarian, and Mohsen Gitizadeh. 2015. "Multi-objective optimal electric power planning in the power system using Gaussian bare-bones imperialist competitive algorithm." Information Sciences 294: 286-304. 
Ghasemi, Mojtaba, Sahand Ghavidel, Shima Rahmani, Alireza Roosta, and Hasan Falah. 2014b. "A novel hybrid algorithm of imperialist competitive algorithm and teaching learning algorithm for optimal power flow problem with non-smooth cost functions." Engineering Applications of Artificial Intelligence 29: 54-69.

Gopalakrishnan, A., A. U. Raghunathan, D. Nikovski, and L. T. Biegler. 2012. "Global optimization of optimal power flow using a branch \& bound algorithm." IEEE Proceedings of the $50^{\text {th }}$ Annual Allerton Conference on Communication, Control, and Computing, Illinois, USA 609-616.

Grimstad, B., and A. Sandnes. 2016. "Global optimization with spline constraints: a new branchand-bound method based on B-splines." Journal of Global Optimization 65 (3): 401-439.

Hansen, E. R., and G. W. Walster. 2005. Global optimization using interval analysis, $2^{\text {nd }}$ edition. New York: Marcel Dekker.

Kocuk, B., S. S. Dey, and Xu. A. Sun. 2016. "Inexactness of SDP relaxation and valid inequalities for optimal power flow." IEEE Transactions on Power Systems 31 (1): 642-651.

Kundur, P. 1994. Power system stability and control. McGraw-Hill, New York, USA.

Lavaei, J., and S. H. Low. 2012. "Zero duality gap in optimal power flow problem." IEEE Transactions on Power Systems 27 (1): 92-107.

Lebbah, Yahia, Claude Michel, and Michel Rueher. 2007. "An efficient and safe framework for solving optimization problems." Journal of Computational and Applied Mathematics 199 (2): $372-377$.

Lesieutre, B. C., D. K. Molzahn, A. R. Borden, and C. L. DeMarco. 2011. "Examining the limits of the application of semidefinite programming to power flow problems." IEEE Proceedings of the $49^{\text {th }}$ Annual Allerton Conference on Communication, Control, and Computing, Illinois, USA 1492-1499.

Li, D., and X. Li. 2016. "Decomposition-based global optimization for optimal design of power distribution systems." $55^{\text {th }}$ IEEE Proceedings of Control and Decision Conference, Las Vegas, USA 3265-3270.

Li, D., and X. Sun. 2010. Nonlinear integer programming. New York: Springer.

Madani, R., M. Ashraphijuo, and J. Lavaei. 2014. User's manual on SDP solver of optimal power flow. University of California, Berkley.

Mohamed, Al-Attar Ali, Yahia S. Mohamed, Ahmed A.M. El-Gaafary, and Ashraf M. Hemeida. 2017. "Optimal power flow using moth swarm algorithm." Electric Power Systems Research 142: 190-206.

Molzahn, D., J. Holzer, B. Lesieutre, and C. DeMarco. 2013. "Implementation of a large-scale optimal power flow solver based on semidefinite programming." IEEE Transactions on Power Systems 28 (4): 3987-3998.

Momoh, J., M. El-Hawary, and R. Adapa. 1999a. "A review of selected optimal power flow literature to 1993. Part I: Nonlinear and quadratic programming approaches." IEEE Transactions on Power Systems 14 (1): 96-104.

Momoh, J., M. El-Hawary, and R. Adapa. 1999b. "A review of selected optimal power flow literature to 1993. Part II: Newton, linear and interior programming." IEEE Transactions on Power Systems 14 (1): 105-111.

Moore, R. E., R. B. Kearfott, and M. J. Cloud. 2009. Introduction to interval analysis. Philadelphia: SIAM.

Nataraj, P. S. V., and M. Arounassalame. 2011. "Constrained global optimization of multivariate polynomials using Bernstein branch and prune algorithm." Journal of Global Optimization 49 (2): $185-212$.

NEOS server. 2018. Available at http://www.neos-server.org/neos/solvers/index.html .

Patil, Bhagyesh V., P. S. V. Nataraj, and Sharad Bhartiya. 2012. "Global optimization of mixed-integer nonlinear (polynomial) programming problems: the Bernstein polynomial approach." Computing 94 (2-4): 325-343.

Patil, B. V., Sampath L. P. M. I., A. Krishnan, K. V. Ling, and H. B. Gooi. 2016. "Examining the Bernstein global optimization approach to optimal power flow problem." AIP Proceedings 1776 (1): 1-6.

Phan, D., and J. Kalagnanam. 2014. "Some efficient methods for solving the security- 
constrained optimal power flow problem." IEEE Transactions on Power Systems 29 (2): 863-872.

Ratschek, H., and J. Rokne. 1988. New computer methods for global optimization. Chichester, England: Ellis Horwood Publishers.

Roa-Sepulveda, C. A., and B. J. Pavez-Lazo. 2003. "A solution to the optimal power flow using simulated annealing." International Journal of Electrical Power Energy Systems 25 (1): $47-57$.

Shaheen, A. M., R. A. El-Sehiemy, and S. M. Farrag. 2016. "Solving multi-objective optimal power flow problem via forced initialised differential evolution algorithm." IET Generation, Transmission Distribution 10 (7): 1634-1647.

Soares, J., T. Sousa, Z. A. Vale, H. Morais, and P. Faria. 2011. "Ant Colony Search algorithm for the optimal power flow problem." IEEE Proceedings of Power and Energy Society General Meeting, Denver, CO 1-8.

Tawarmalani, M., and N. V. Sahinidis. 2002. Convexification and global optimization in continuous and mixed-integer nonlinear programming: theory, algorithms, software, and applications (Nonconvex optimization and its applications). Dordrecht: Kluwer Academic Publishers.

Tawarmalani, M., and N. V. Sahinidis. 2005. "A polyhedral branch-and-cut approach to global optimization." Mathematical Programming 103 (2): 225-249.

The Mathworks Inc., MATLAB version 8.3 (R2014a). Natick, MA, 2014.

Todorovski, M., and D. Rajicic. 2006. "An initialization procedure in solving optimal power flow by genetic algorithm." IEEE Transactions on Power Systems 21 (2): 480 487.

Vaidyanathan, R., and M. El-Halwagi. 1996. Global optimization of nonconvex MINLP's by interval analysis. In: Global Optimization in Engineering Design. New York, pp. 175-193: Springer.

Vaisakh, K., and L.R. Srinivas. 2011. "Adaptive PSODV algorithm for OPF with nonsmooth cost functions and statistical analysis." Simulation Modelling Practice and Theory 19 (9): 1824-1846.

Zhijun, Q., Y. Hou, and Y. Chen. 2015. "Convex envelopes of optimal power flow with branch flow model in rectangular form." IEEE Proceedings of Power and Energy Society General Meeting, Denver, CO 1-5.

Zimmerman, R. D., C. E. Murillo-Sanchez, and R. J. Thomas. 2011. "MATPOWER: Steady-state operations, planning, and analysis tools for power systems research and education." IEEE Transactions on Power Systems 26 (1): 12-19. 